\title{
The effect of heteroplasmy on cytoplasmic incompatibility in transplasmic lines of Drosophila simulans showing a complete replacement of the mitochondrial DNA
}

\author{
LOREDANA NIGRO \\ Department of Biology, University of Padova, via Trieste 75, 35121 Padova, Italy
}

\begin{abstract}
Unidirectional cytoplasmic incompatibility has been observed in crosses between different strains of Drosophila simulans when the males, but not the females, involved in the crosses are infected with intracellular rickettsia-like microorganisms. In contrast to what is known about this system an unexpected partial incompatibility has been observed between two infected strains. Transplasmic lines have been constructed in the laboratory to investigate this phenomenon. The injection of a foreign infected cytoplasm into a strain which apparently shows the same kind of bacterial infection causes an incompatibility in crosses between injected heteroplasmic males, but not injected females, of the same strain. These findings suggest that several crossing types exist within the Drosophila simulans incompatibility system. The complete replacement of the original mitochondrial DNA observed in some of the transplasmic lines made it possible to analyse and exclude any link between incompatibility and mitochondrial genomes.
\end{abstract}

Keywords: cytoplasmic incompatibility, Drosophila stimulans, rickettsia-like organisms, transplasmic lines.

\section{Introduction}

The existence of unidirectional cytoplasmic incompatibility has recently been discovered in crosses among different populations of Drosophila simulans (Hoffman et al., 1986; Hoffman \& Turelli, 1988; Nigro \& Prout, 1990) and Drosophila melanogaster (Hoffman, 1988). When a male of one strain is crossed to a female of another strain the cross is either sterile or gives few progeny. The number of $F_{1}$ progeny in these crosses is always significantly reduced compared either with the reciprocal cross or with crosses within the parental strains. The involvement of extranuclear microorganisms has been the most favoured explanation for such incompatibility although the mechanism is not as yet understood.

Analysis in the electron microscope of the strains involved in the crosses shows that, in the case of unidirectional incompatibility, the males are always heavily infected with rickettsia-like intracytoplasmic prokaryotes (possibly Wolbachia pipientis) while the females are not (Louis \& Nigro, 1989; Binnington \& Hoffman, 1989). Similar analyses of the progeny obtained in both reciprocal crosses showed the mater- nal inheritance of the bacterial infection. This provides supporting evidence for the involvement of bacteria since related incompatibility systems are known to be maternally inherited (Hoffmann \& Turelli, 1988; Nigro \& Louis, 1990). The link observed between bacterial infection and incompatibility makes it possible to screen different strains by using infected males as a 'tester'. The fertility or sterility of any cross made between males of this strain and females of any other strain indicates whether or not the strain tested is likely to be infected. Here I describe the existence of partial incompatibility between two strains that, after analysis in the electron microscope, seem to be infected by morphologically indistinguishable bacteria.

The existence of partial incompatibility between two infected strains has never been described in Drosophila and cannot be explained in the light of the incompatibility system as described above. These findings suggest the coexistence of different crossing types in Drosophila, as has already been described for a similar phenomenon in mosquitoes (Subbarao, 1982). This hypothesis has been analysed using experimental transplasmic flies obtained by injecting the cytoplasm of one infected strain into the eggs of another. 


\section{Materials and methods}

The three strains used for this work come from different locations. Two are from Italy and have already been analysed for the incompatibility system. One of them (strain C) is infected and the other (strain P) is not. The third strain (strain $M$ ) was kindly provided by Michel Solignac and comes from the Mont d'Ambre (Madagascar). It too is infected.

The transplasmic line $(\mathrm{Mj})$ was obtained by injecting, before pole cell formation, cytoplasm from the eggs of strain $C$ into the posterior end of eggs of strain $M$ according to the technique described by Santamaria (1987). Strains C and M belong to two different morphs with respect to their mitochondrial DNA (mtDNA). Strain C is type II while strain M is type III (Solignac et al., 1986). Apart from other differences, their mtDNA patterns differ after digestion with the endonuclease HpaII and this makes it possible to test the success of the cytoplasm transfer by looking for the existence of heteroplasmic state due to the presence in the recipient cytoplasm of mitochondria received from the donor.

Several different sublines were established from an injected heteroplasmic female. Some of them remained heteroplasmic, whereas some reverted to the original mitochondrial state and some switched to the mitochondrial morph of the donor strain. All strains were cultured on standard medium made with sucrose, agar and brewer's yeast with nipagin as a mould inhibitor.

Incompatibility among the strains was tested by allowing single pairs of 3-day-old males and females to breed in separate vials with 20 replicates per cross. After two weeks the progeny number obtained was counted and compared by Kruskall-Wallis and Wilcoxon tests (Sokal \& Rohlf, 1981). The incompatibility within injected and not-injected individuals of the $M$ strain was tested by crossing females and males of the

Table 1 Means and standard deviations based on 15-20 replicate vials

\begin{tabular}{|c|c|c|c|c|c|c|}
\hline & \multicolumn{3}{|l|}{ Crosses } & \multicolumn{2}{|c|}{ Progeny } & \multirow[b]{2}{*}{ Significance* } \\
\hline & ○ & & 0 & $\bar{x}$ & (SD) & \\
\hline 1 & $\mathrm{C}$ & $x$ & $\mathrm{C}$ & 17.86 & $(5.56)$ & \\
\hline 2 & $P$ & $x$ & $P$ & 19.43 & $(8.10)$ & \\
\hline 3 & $\mathbf{M}$ & $x$ & M & 22.28 & $(7.93)$ & \\
\hline 4 & $\mathrm{C}$ & $x$ & $\mathrm{P}$ & 17.25 & (4.99) & \\
\hline 5 & $P$ & $x$ & $\mathrm{C}$ & 3.32 & $(3.60)$ & \\
\hline 6 & $P$ & $x$ & $\mathbf{M}$ & 0 & & \\
\hline 7 & M & $x$ & $\mathrm{P}$ & 19.33 & $(4.13)$ & $* * *$ \\
\hline 8 & M & $x$ & $\mathrm{~F}_{1}\left(\mathrm{OM} \times \sigma^{\prime \prime} \mathrm{P}\right)$ & 16 & $(2.75)$ & \\
\hline 9 & $P$ & $x$ & $\mathrm{~F}_{1}\left(\$ \mathbf{M} \times \sigma^{\prime} \mathbf{P}\right)$ & 0 & $(0.32)$ & \\
\hline 10 & 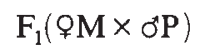 & $x$ & $\mathrm{P}$ & 18.8 & $(5.00)$ & \\
\hline 11 & $\mathrm{~F}_{1}(\% \mathrm{M} \times \overbrace{}^{*} \mathrm{P})$ & $x$ & M & 21.9 & $(7.58)$ & \\
\hline 12 & $\mathrm{M}$ & $x$ & $\mathrm{~F}_{1}\left(\mathrm{PP} \times \mathrm{O}^{\prime \prime} \mathrm{M}\right)$ & 20.12 & $(7.44)$ & \\
\hline 13 & $P$ & $x$ & $\mathrm{~F}_{1}\left(\mathrm{QP} \times \mathrm{O}^{\top} \mathrm{M}\right)$ & 21.8 & $(3.71)$ & \\
\hline 14 & $\mathrm{~F}_{1}\left(\wp \mathrm{P} \times \sigma^{\prime} \mathbf{M}\right)$ & $x$ & $\mathrm{P}$ & 25.5 & $(7.67)$ & \\
\hline 15 & $\mathrm{~F}_{1}\left(\% \mathrm{P} \times \sigma^{\prime} \mathrm{M}\right)$ & $x$ & M & 1.23 & $(2.05)$ & \\
\hline
\end{tabular}

*Probability for Kruskall-Wallis test.

*** $=P<0.001$.

\begin{tabular}{rl} 
Comparisons & Signifiance level for Wilcoxon tests \\
\hline 1 vs. 4 & ns \\
2 vs. 5 & $* * *$ \\
2 vs. 6 & $* * *$ \\
3 vs. 7 & ns \\
8 vs. 12 & ns \\
9 vs. 13 & $* * *$ \\
10 vs. 11 & ns \\
14 vs. 15 & $* * *$ \\
\hline
\end{tabular}


original strain to males and females of the sublines established from the heteroplasmic $\mathrm{Mj}$ female. The effect of the injection on the incompatibility system was also tested by crossing the injected males to females of the $\mathrm{C}$ and $\mathrm{P}$ strains.

To test whether the males involved in the crosses were still heteroplasmic and whether this state had any effect on the incompatibility system, the mtDNA of each single male was extracted according to the method of Coen et al. (1982), digested with the endonuclease HpaII, run on 0.8 per cent agarose gels in TBE buffer, transferred by Southern blotting and then hybridized to a mtDNA probe labelled with ${ }^{32} \mathrm{P}$ by nick translation. The highly purified mtDNA used as probe was extracted from unfertilized eggs according to the procedure described in Solignac \& Monnerot (1986).

On this basis it has been possible to characterize each single male either as belonging to one of the two original types or as being heteroplasmic.

\section{Results}

The results of crosses performed between the three strains $\mathrm{P}, \mathrm{C}$ and $\mathrm{M}$ are shown in Table 1 . They confirm the unidirectional incompatibility between males of $\mathrm{C}$ strain and females of $P$ strain and show the existence of a similar incompatibility between the $P$ strain and the $M$ strain. The number of progeny obtained in crosses between $P$ female and either $\mathrm{C}$ and $\mathrm{M}$ males was in fact always significantly different from the number obtained in the reciprocal crosses or within the parental strains.

The inheritance of the incompatibility system between the $\mathrm{M}$ and $\mathrm{P}$ strains has been tested by backcrossing reciprocal $F_{1}$ flies obtained from the reciprocal crosses to the parental strains. The results shown in Table 1 confirmed the maternal inheritance of this system over one generation in agreement with what has already been seen between the $\mathrm{C}$ and $\mathrm{P}$ strains. Given that the analysis in the electron microscope had shown that both the $\mathrm{C}$ strain and $\mathrm{M}$ strain, but not the $\mathrm{P}$ strain, were infected by a rickettsia-like microorganism, these results are in agreement with what is already known about the incompatibility system in Drosophila simulans.

In contrast, the results obtained in the crosses between the two infected strains are not consistent with this system, as described above (Table 2). Despite the fact that the $\mathrm{M}$ and $\mathrm{C}$ strains are infected by morphologically indistinguishable bacteria, both reciprocal crosses show the existence of partial incompatibility, with a reduction in fertility. To try to understand the origin of this partial incompatibility the progeny of several sublines established from the heteroplasmic $\mathrm{Mj}$ line have been crossed to the original strain $\mathrm{M}$. The
Table 2 Means and standard deviations based on 15-20 replicate vials

\begin{tabular}{|c|c|c|c|c|c|c|}
\hline & \multicolumn{3}{|c|}{ Crosses } & \multicolumn{2}{|c|}{ Progeny } & \multirow[b]{2}{*}{ Significance } \\
\hline & q & & ठ" & $\bar{x}$ & (SD) & \\
\hline 1 & $\mathbf{M}$ & $x$ & M & 23.72 & $(10.32)$ & \\
\hline 2 & $\mathrm{C}$ & $x$ & $\mathrm{C}$ & 18.83 & $(6.57)$ & \\
\hline 3 & $\mathbf{M}$ & $x$ & $\mathrm{C}$ & 11.59 & $(16.92)$ & \\
\hline 4 & $\mathrm{C}$ & $x$ & $\mathbf{M}$ & 13.42 & $(10.40)$ & \\
\hline 5 & $\mathbf{M}$ & $x$ & $\mathrm{Mj}^{*}$ & 0.5 & $(1.41)$ & $* * *$ \\
\hline 6 & $\mathbf{M}$ & $x$ & $\mathrm{Mj}$ & 10 & $(10.30)$ & \\
\hline 7 & $\mathrm{Mj}^{*}$ & $x$ & $\mathrm{M}$ & 14.3 & $(14.54)$ & \\
\hline 8 & $\mathrm{Mj}$ & $x$ & $\mathbf{M}$ & 18.69 & $(16.40)$ & \\
\hline 9 & $\mathrm{C}$ & $x$ & $\mathrm{Mj}^{*}$ & 3.8 & $(5.50)$ & \\
\hline 10 & $\mathrm{P}$ & $x$ & $\mathrm{Mj}$ & 0.25 & $(0.70)$ & \\
\hline
\end{tabular}

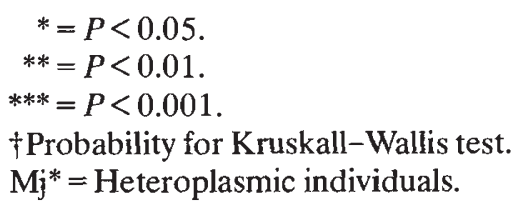

Comparisons Significance level for Wilcoxon tests

\begin{tabular}{ll}
\hline 1 vs. 2 & ns \\
1 vs. 3 & $* *$ \\
1 vs. 4 & $* *$ \\
2 vs. 3 & $*$ \\
2 vs. 4 & $*$ \\
3 vs. 4 & ns \\
1 vs. 5 & $* * *$ \\
1 vs. 6 & ns \\
1 vs. 7 & $* *$ \\
1 vs. 8 & ns \\
4 vs. 9 & $*$ \\
\hline
\end{tabular}

progeny numbers obtained in reciprocal crosses between the $\mathrm{M}$ and $\mathrm{Mj}$ lines have been analysed and compared both to the crosses within parental strains and to crosses between $\mathrm{Mj}$ males and $\mathrm{C}$ females (Table 2 ). The pattern obtained after digestion of the mtDNA extracted from each single male involved in the crosses is shown in Fig. 1. Some of the males are still heteroplasmic while the others show fixation either of the original mtDNA (type III) or show a complete replacement of the original mtDNA with the mtDNA of the donor (type II).

In the crosses between $\mathrm{Mj}$ males and $\mathrm{M}$ or $\mathrm{C}$ females an incompatibility has been detected when the injected male is still heteroplasmic. This suggests the presence and interaction (within these males) of different cytoplasmic factors, originally present in the two strains. In the reciprocal cross and in the crosses where the males are not heteroplasmic, the level of fertility is either 


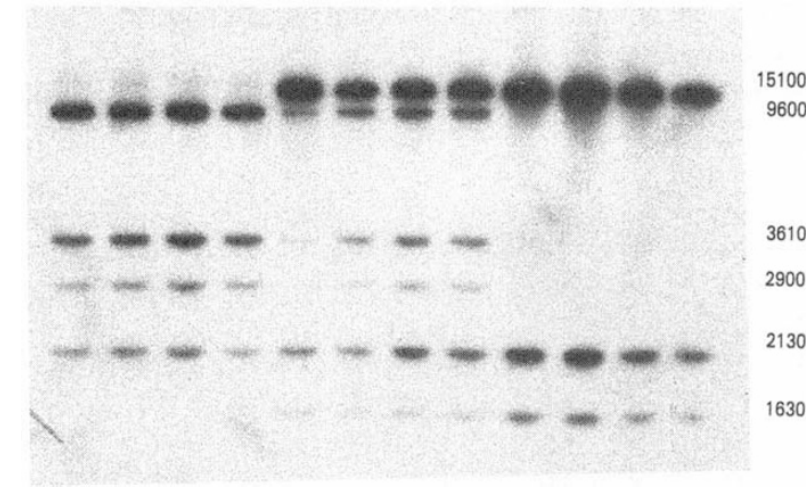

Fig. 1 Autoradiography of a Southern blot of mitochondrial DNA extracted from single males. Lane 1-4, pure siII sublines; lanes 9-12, pure siIII sublines; lanes 5-8, heteroplasmic siII/siIII sublines.

reduced, as it was in the reciprocal crosses between the original strains, or is similar to that observed within the original strains.

The complete replacement of the mtDNA in some of the lines made it possible to analyse and to exclude any involvement of the mitochondrial DNA in the incompatibility system. No differences were found (in terms of incompatibility) in the crosses between $M$ females and $\mathrm{Mj}$ males according to the kind of mtDNA (type II or type III) fixed in the male.

Sterility was, in fact, detected in the crosses with males showing fixation either of the type II or the type III mtDNA. The persistence of the bacterial infection in the line $\mathrm{Mj}$ was checked and confirmed by electron microscope (C. Louis, personal communication) as was the persistence of the related phenomenon of cytoplasmic incompatibility by crossing $\mathrm{Mj}$ males to $\mathrm{P}$ females (Table 2).

\section{Discussion}

The results obtained in this experiment confirm what is already known for the incompatibility system in Drosophila with respect to the involvement of a Wolbachialike prokaryote (Binnington \& Hoffman, 1989; Louis \& Nigro, 1989; Nigro \& Louis, 1990).

A similar phenomenon of cytoplasmic incompatibility, either unidirectional or bidirectional, has been known and intensively studied for many years in mosquitoes. There is substantial variability in the factors controlling this phenomenon (reviewed by Magnin \& Pasteur, 1987) and 17 different crossing types have been discovered among 37 strains (Subbarao, 1982).

The partial incompatibility reported here between two infected strains of Drosophila simulans can be explained if we postulate the existence of different crossing types as seen in mosquitoes. If this is so the incompatibility of a strain cannot be strictly defined since it would show substantial variability according to the partner in the cross. The results presented here suggest that a cytoplasmic polymorphism is present in natural populations of Drosophila simulans. Analysis in the electron microscope of the $\mathrm{C}$ and $\mathrm{M}$ strains seems to show that they are infected by morphologically indistinguishable bacteria. These may well be Wolbachia pipientis, reportedly one of the causative agents of incompatibility in mosquitoes.

This morphological similarity, of course, does not exclude the possibility that the variation results from differences in the infecting bacteria. The interaction between insect and bacteria is subject to evolution and this may lead to differences in the mechanisms affecting the reproductive potential of the insect. Experimental support for this hypothesis would require a more certain classification of the bacteria and a study of the bacterial genomes. This may be difficult, however, because of the problems associated with the culture of intracellular prokaryotes such as rickettsia. In fact relatively little is known about the biochemistry and molecular biology of such organisms.

Different hypotheses have been proposed to explain the phenomenon of cytoplasmic incompatibility but up to now there is no direct evidence for any of them. In any case it seems likely that, at least in mosquitoes, the phenomenon of incompatibility involves the action not only of a symbiotic prokaryote but also of other cytoplasmic factors which result from the presence of the microorganism. Their presence or absence in the oocytes could also explain the observed segregation of the cytoplasmic determinants. The phenomenon of segregation was first observed in mosquitoes (Barr, 1980) where individual females gave rise to offspring exhibiting great heterogeneity of crossing compatibility. Similar segregation, apparently transient, has been reported in Drosophila by Hoffman \& Turelli (1988) in just one line, but in this case a rare case of paternal transmission was apparently involved.

The results presented here cannot provide conclusive evidence but can suggest the existence in Drosophila of different crossing types in the $\mathrm{M}$ and $\mathrm{C}$ strains. This would account both for the partial incompatibility observed between them and for the incompatibility observed between the $M$ female and the heteroplasmic $\mathrm{Mj}$ males. Both situations would result from an interaction of the different cytoplasmic components which affected the level of expression of incompatibility between the strains. The expressivity of these cytoplasmic factors could change according to the particular cytoplasmic system to which they are co-adapted. In mosquitoes it has been shown that there is a relationship between teratological growth pattern and the 
degree of heterozygosity of different cytoplasmic sterility factors (French, 1978).

Further experiments are needed to confirm and eventually to elucidate this hypothesis. Ideally, the experiment should be repeated with a strain showing a completely different type of incompatibility in order to analyse the mode of interaction between the factors involved. In addition, it would be necessary to characterize at the molecular level the rickettsia and/or the cytoplasmic factors involved in this phenomenon and follow their segregation.

The complete replacement of the mtDNA observed in some of the transplasmic strains is an interesting result per se. As well as excluding any link between mitochondrial genomes and the incompatibility system, the construction of such transplasmic flies offers the opportunity of planning experiments to analyse the existence of intracellular selection of different endogenous mtDNA in different nuclear backgrounds. As such, the technique would provide a very useful tool to study the reciprocal compatibility of nuclear and mitochondrial genomes.

\section{Acknowledgements}

I thank Eric de Stordeur for assistance in the injections and T. Prout, M. Solignac and M. Turelli for helpful comments on the manuscript.

This work was supported by CNR grant N.880200904.

\section{References}

3ARR, A. R. 1980. Cytoplasmic incompatibility in natural populations of a mosquito, Culex pipiens L., Nature, 283, 71-72.

BINNINGTON, K. C. AND HOFFMAN, A. A. 1989. Wolbachia-like organisms and cytoplasmic incompatibility in Drosophila simulans. J. Invert. Pathol., 54, 344-352.

COEN, E. S., THODAY, J. N. AND DOVER, G. 1982. Rate of turnover of structural variants in the rDNA gene family of Drosophila melanogaster. Nature, 295, 564-568.

FRENCH, w. L. 1978. Genetic and phenogenetic studies on the dynamic nature of the cytoplasmic inheritance system in Culex pipients. Genetics, 88, 447-455.

HOFFMAN, A. A., TURELLI, M. AND SIMMONS, G. M. 1986. Unidirectional incompatibility between populations of Drosophila simulans. Evolution, 40, 692-701.

HOFFMAN, A. A. 1988. Partial cytoplasmic incompatibility between Australian populations of Drosophila melanogaster. Entomol. exp. appl., 48, 61-67.

HOFFMAN, A. A. AND TURELLI, M. 1988. Unidirectional incompatibility in Drosophila simulans: Inheritance, geographic variation and fitness effects. Genetics, 119, 435-444.

LOUIS, C. AND NIGRO, L. 1989. Ultrastructural evidence of Wolbachia Rickettsiales in Drosophila simulans and their relationship with unidirectional cross-incompatibility. $J$. Invert. Pathol., 54, 34-44.

MAGNIN, M. AND PASTEUR, N. 1987. Incompatibilities cytoplasmiques dans le complexe Culex pipiens. Une revue. Cah. ORSTOM, 1, 45-53.

NIGRO, L. AND PROUT, T. 1990. Is there selection on RFLP differences in mtDNA? Genetics, 125, 551-555.

NIGRO, L. AND LouIs, C. 1990. Wolbachia endosymbiotes as putative agents of unidirectional cross-incompatibility in Drosophila. In Endocytobiology (in press).

SANTAMARIA, P. 1987. Injecting eggs. In Roberts, D. B., Drosophila: a practical approach. IRL Press, Oxford, 159-173.

SOLIGNAC, M., MONNEROT, M. AND MOUNOLOU, J. C. 1986. Mitochondrial DNA evolution in the melanogaster species subgroup of Drosophila. J. molec. Evol., 23, 53-60.

SOLIGNAC, M. AND MONNEROT, M. 1986. Race formation, speciation and introgression within the three homosequential species Drosophila simulans, D. mauritiana and $D$. sechellia inferred from their mitochondrial DNA analysis. Evolution, 40, 531-539.

SOKAL, R. R. AND ROHLF, F. J. 1981. Biometry, 2nd edn, Freeman, San Francisco, CA.

subBaraO, s. K. 1982. Cytoplasmic incompatibility in mosquitoes. In Steiner, W. W. M., Tabachnick, W. J., Rai, K. S. and Narang, S., Recent Developments in the Genetics of Insect Disease Vectors. Stipes Publishing Company, Champaign, Illinois, 313-342. 